\title{
Physicians' behavior following changes in LDL cholesterol target goals
}

\author{
Shlomo Vinker ${ }^{1,2}$, Haim Bitterman ${ }^{1}$, Doron Comaneshter ${ }^{1}$ and Arnon D Cohen ${ }^{1,3^{*}}$
}

\begin{abstract}
Background: In 01/2011 Clalit Health Services (CHS), changed the LDL-Cholesterol target definitions in its quality indicators program, from a universal target to values stratified by risk assessment based on ATP III criteria. The objective of this study is to evaluate the effect of this change on achievement of LDL-C targets and on physicians' prescriptions of statins.

Study Design: A descriptive study based on administrative dataset 06/2010-06/2012.

Methods: Setting: CHS, The largest health maintenance organization in Israel that insures above 4,000,000 beneficiaries.

Patients: Patients who had been in the same risk group throughout the study period.

Measurements: Attainment of targets for LDL-C and purchases of statins prior to, and following, implementation of the guidelines in the CHS quality indicators program.

Results: 433,662 patients remained in the same risk groups throughout the study period; $55.8 \%$ were women; the average age was $53.0 \pm 10.3$ years; $63.9 \%, 13.4 \%$, and $22.7 \%$ were at low, medium, and high risk respectively. After implementation, the proportion of patients reaching LDL-C targets increased in all risk groups: from $58.6 \%$ to $61.6 \%$, from $55.1 \%$ to $61.1 \%$, and from $44.5 \%$ to $49.0 \%$, in low, medium, and high risk groups respectively $(p<0.001)$. The proportion of patients treated with potent statins increased in all risk groups; from $3.4 \%$ to $5.6 \%$, from $6.7 \%$ to $10.3 \%$, and from $14.5 \%$ to $20.3 \%$ respectively $(p<0.001)$.
\end{abstract}

Conclusion: The risk stratification approach as a basis for the quality indicators program was implemented and better achievement of target LDL-C levels ensued. We suggest that implementation of quality indicators that are consistent with the current literature can lead to improvements that exceeds temporal trends.

Keywords: Quality indicators, Clinical guidelines, Hypercholesterolemia, Statins

\section{Background}

Healthcare services operate in increasingly complex environments, characterized by growing specialization, fragmentation of medical care, and soaring costs that do not always reflect healthcare value. Healthcare quality assurance programs aim to enhance the extent to which healthcare services achieve desired outcomes, according to the prevailing professional literature. An increasing number of pay-for-performance systems employ financial incentives to achieve quality measures that are focused on

\footnotetext{
*Correspondence: arcohen@clalit.org.il

${ }^{1}$ Chief Physician Office, Central Headquarter, Clalit Health Services, Tel Aviv, Israel

${ }^{3}$ Siaal Family Medicine and Primary Care Research Center, Faculty of Health Sciences, Ben-Gurion University of the Negev, Beer-Sheva, Israel

Full list of author information is available at the end of the article
}

health outcomes, rather than on process-of-care [1]. However, the pay-for-performance model has been reported to yield only modest [2] and even negligible and inconsistent improvements in healthcare quality [3,4], and the costs of such programs have yet to be assessed [5].

The National Cholesterol Education Program Adult Treatment Panel III (ATP III) guidelines serves as the benchmark for the assessment of the quality of treatment of hyperlipidemia [6]. Published studies have demonstrated low adherence to them, despite assessment of their relative cost-effectiveness [7]. For example, Barham et al. found that the challenge facing implementation of ATP III guidelines is much greater for intermediate- and high-risk patients than for low-risk patients. [8]. Lee et al. also noted that the rate of achieving target LDL-C levels was lower in 
patients at higher risk for cardiovascular disease [9]. Computerized clinical decision support systems, including recommendations tailored to patient characteristics, have been shown to mildly increase physician adherence to ATP III guidelines [10], but not to exert a statistically significant effect on LDL-C target achievement [11]. Numerous barriers to guideline adherence by physicians have been identified [12].

All Clalit Health Services (CHS) institutions are required to measure and follow their performance in core clinical areas of operation including both processes of care and healthcare outcomes. At the beginning of every calendar year, $\mathrm{CHS}$ publishes its quality indicator program for the upcoming year, with details of targeted changes and the rationale behind them. Guidelines and performance updates are transferred electronically to all CHS physicians and nurses on a monthly basis.

In 2006, CHS set an ambitious target of LDL cholesterol $<100 \mathrm{mg} / \mathrm{dl}$, to be reached by $90 \%$ of patients who are after therapeutic cardiac catheterization or coronary artery bypass grafts, $65 \%$ of patients with diabetes, and $20 \%$ of the remaining individuals with hyperlipidemia. In January 2011, the CHS program of quality indicators was revised and the ATP III guidelines for the management of dyslipidemia of patients without diabetes or active ischemic heart disease were used as a guide for risk stratification [6]. Accordingly, all members of CHS without diabetes or ischemic heart disease were stratified by risk assessment and the automatic reminders for $\mathrm{CHS}$ physicians were changed accordingly.

The aim of the current study was to evaluate the longitudinal effect of these changes on achievement of LDL-C targets, and on the delivery of care, as measured by medication purchases.

\section{Methods}

This is a descriptive study based on an administrative dataset of purchases of statins and of attainment of risk stratified targets for LDL-C prior to, and following, implementation of ATP III based guidelines in CHS quality indicators program.

\section{Data sources}

Data were accessed from the CHS data warehouse. CHS is the largest health maintenance organization in Israel, insuring and providing healthcare to more than $50 \%$ of Israel's population (more than 4,000,000 beneficiaries). Every person insured by CHS is under the care of a primary care physician (PCP), either a family physician or a pediatrician. Patients only see the PCP to whom they are assigned (except for when their physician is on vacation, when they are out of town, or when there is urgency and their physician is not available). For each visit to a different PCP, a special administrative certificate of approval is needed and the peer physician is instructed to provide only "first aid". Hence, provision of primary care in CHS is characterized by a high level of continuity [13].

The CHS information system is comprehensive, comprising socio-demographic data; information on the utilization of healthcare services, drug purchases, laboratory and imaging tests, and a wide-scale registry of chronic diagnoses [14]. The epidemiology unit of the CHS maintains a central comprehensive chronic diseases registry. This registry is continuously updated, based on an algorithm integrating all available data (hospitalization discharge diagnoses, chronic diagnoses in the PCP electronic medical record, laboratory test results, drug purchases and other sources). CHS's registry of patients with chronic diseases serves as the foundation for calculations of healthcare quality indicators for several chronic conditions such as hypertension, diabetes, and ischemic heart disease.

All community pharmacies operated by CHS are computerized and report to a central data repository. All prescriptions of statins that were filled by CHS members between June 1, 2010 and June 31, 2012 were documented. CHS dispenses medications with nominal and almost equal co-payment, which ensures that all prescriptions are documented and that drug selection is not influenced by financial considerations. The formulary choices offered did not change over the study period, as generic atorvastatin had been introduced on $07 / 2010$ and generic rosuvastatin on 06/2010.

LDL-c tests were performed in the CHS central laboratories, using the same techniques during the study period.

\section{Patient population}

The CHS chronic disease registry identifies people with diabetes and after therapeutic cardiac catheterization or coronary artery bypass grafts, for whom the LDL-C target is $<100 \mathrm{mg} \%$. These patients have different quality indicator sets. For all other patients with a diagnosis of hyperlipidemia in CHS registry, cardiovascular risk was assessed based on the ATP III. The following variables were used to assess risk: age and gender, smoking status (patients for whom smoking status was missing were recorded as "non-smokers"), hypertension, cardiovascular diseases (ischemic heart disease without therapeutic cardiac catheterization or coronary artery bypass grafts, peripheral vascular disease, state after cerebrovascular accident), and most recent HDL level (on June 2010 and again on June 2012). We did not have data on family history of cardio-vascular diseases.

We stratified the risk groups according to a modification to the ATP III:

- Patients with $0-1$ risk factors were classified as "low risk" with LDL-C target of $<160 \mathrm{mg} / \mathrm{dl}$. 
- Patients with 2 risk factors were classified as "moderate risk" with LDL-C target of $<130 \mathrm{mg} / \mathrm{dl}$.

- Patients with $>=3$ risk factors or with proven cardiovascular diseases were classified as "high risk" with LDL-C target of $<100 \mathrm{mg} / \mathrm{dl}$.

510,166 patients were included in the QI program in $06 / 2010$, of them 76,504 changed risk group during the study period and 433,622 were in the same risk group in $06 / 2012$. Only patients who had been in the same risk group throughout the study period (June 2010 to June 2012) were included in the analysis.

Demographic data included: Age, gender and socioeconomic status (SES); low SES was defined as exemption from social security payments.

\section{Main outcome measures}

1. The proportion of patients who reached the LDL-C target value for their risk category.

2. The proportion of patients using potent statins (Atorvastatin and Rosuvastatin) and first- line statins (all other statins) in each risk category.

Outcomes were assessed every six months, starting six months prior to implementation of the stratified approach, and continuing until 18 months after implementation. As it was a retrospective study we evaluated at each point only patients with a valid LDL-C test (a valid test had been defined as a test done in the last 12 months).

The study was approved by Clalit Health Services ethics committee. The committee states that there is no need of informed consent of study subjects.

\section{Statistical analysis}

Comparison between three groups of patients (low, medium, and high risk) with regard to demographic parameters (gender, age, etc.) was performed using oneway analysis of variance (ANOVA) and Chi-square tests, as applicable.

ANOVA with repeated measures (over time) was performed to assess the time trend in the various outcome parameters (prescription of statins, achievement of LDL targets). Contrast analysis was used to compare successive time points vs. baseline.

The statistical significance level was set to 0.05 and the SPSS for Windows software, version 19.0 (Chicago, IL), was used for the analysis.

\section{Results}

The study population included all the 433,362 patients of CHS who remained in the same risk group throughout the study period. Their socio-demographic characteristics are summarized in Table 1. Patients in the low risk category tended to be younger $(\mathrm{p}<0.001)$, and a larger proportion were females $(\mathrm{p}<0.001)$. Patient risk factors are summarized in Table 2.

Figure 1 depicts the trend of target LDL-C achievement according to risk categories. At the beginning of the program target LDL-C achievement was $46.0 \%, 55.1 \%$ and $58.7 \%$ in the high, medium and low risk groups respectively. Small changes (and even a decline) were observed in the first six months after launching the program. Changes became more prominent and significant one year after the introduction of the new stratified targets. At the end of 18 months follow-up, target LDL-C achievement was significantly higher: $49.0 \%, 61.1 \%$ and $61.6 \%$ in the high, medium and low risk groups, respectively.

Figures 2 and 3 demonstrate the secular relationship of first line and potent statin utilization, respectively. For all risk categories there was a significant continual decline in the use of first-line statins and a parallel increase in the percent of individuals on potent statins. The prescription of first- line statins declined from $31.0 \%$ to $26.0 \%$, from $38.4 \%$ to $34.1 \%$, and from $20.4 \%$ to $18.2 \%$ in the high, medium and low risk groups respectively. The prescription of potent statins increased from $16.4 \%$ to $20.3 \%$, from $7.7 \%$ to $10.3 \%$, and from $4.0 \%$ to $5.6 \%$ in the high, medium and low risk groups respectively. The magnitude of the change was greater for higher risk categories. Changes in prescription habits, as reflected by statin purchases, preceded changes in achieving LDL targets.

\section{Discussion}

This study showed that the rate of achievement of target LDL-C decreased during the first 6 months after the implementation of the stratified approach, yet increased

Table 1 Sociodemographic characteristics of the study population

\begin{tabular}{|c|c|c|c|c|c|}
\hline & Total & Low risk & Moderate risk & High risk & p value ${ }^{a}$ \\
\hline Number of individuals & 433,662 & 277,234 & 58,214 & 98,214 & \\
\hline$\%$ of the cohort & $100 \%$ & $63.9 \%$ & $13.4 \%$ & $22.7 \%$ & \\
\hline Age - mean (SD) & $53.0(10.3)$ & $49.2(12.7)$ & $60.4(7.8)$ & $59.2(10.3)$ & $<0.001$ \\
\hline Males (\%) & $44.2 \%$ & $34.2 \%$ & $52.3 \%$ & $78.7 \%$ & $<0.001$ \\
\hline Low socioeconomic status (\%) & $34.9 \%$ & $34.8 \%$ & $32.3 \%$ & $36.7 \%$ & $<0.001$ \\
\hline
\end{tabular}

${ }^{a}$ For difference between the three groups. 
Table 2 Risk factors of participants according to ATPIII

\begin{tabular}{lllll}
\hline & Total & Low risk & Moderate risk & High risk \\
\hline Number of individuals & 433,662 & 277,234 & 58,214 & 98,214 \\
Male (> = age 45 years) & $140,965(32.5 \%)$ & $50,264(18.1 \%)$ & $29,185(50.1 \%)$ & $61,516(62.6 \%)$ \\
Female (> = age 55 years) & $127,264(29.3 \%)$ & $76,815(27.7 \%)$ & $26,721(45.9 \%)$ & $23,728(24.2 \%)$ \\
Current smoking & 76,621 & 42,933 & 8,710 & 24,978 \\
Low HDL cholesterol & 56,290 & 15,650 & 10,614 & 30,026 \\
Hypertension & 125,128 & 22,410 & 45,631 & 57,087 \\
Ischemic heart disease & 59,931 & 0 & 0 & 59,931 \\
State after cerebrovascular accident & 21,864 & 0 & 0 & 21,864 \\
Peripheral vascular disease & 10,436 & 0 & 0 & 10,436 \\
High HDL cholesterol & 80,789 & 69,236 & 1,181 & 10,372 \\
\hline
\end{tabular}

${ }^{\mathrm{a}} \mathrm{High} \mathrm{HDL}$ cholesterol - is a protective factor (in patients with high HDL cholesterol the score of risk factors is subtracted by one).

throughout the subsequent year. Purchases of first-line statins decreased and purchases of potent statins increased in a complementary manner, starting from six months after implementation of stratified quality indicators based on ATP III guidelines. This was true for individuals in all three cardiovascular risk categories, and continued throughout the 18 month study period. This temporal change in process of care and patient outcomes may be partially explained by the influence of the healthcare policy change on physicians' clinical behavior [15]. Financial incentives were not awarded, nor immediate health benefit conferred. Thus, physician recognition of the value of quality measures and confidence in their validity might be the prime factors determining their improved adherence.

More than $10 \%$ of the total number of beneficiaries of CHS comprised the population of the current study. Treatment of chronic conditions, such as hyperlipidemia,

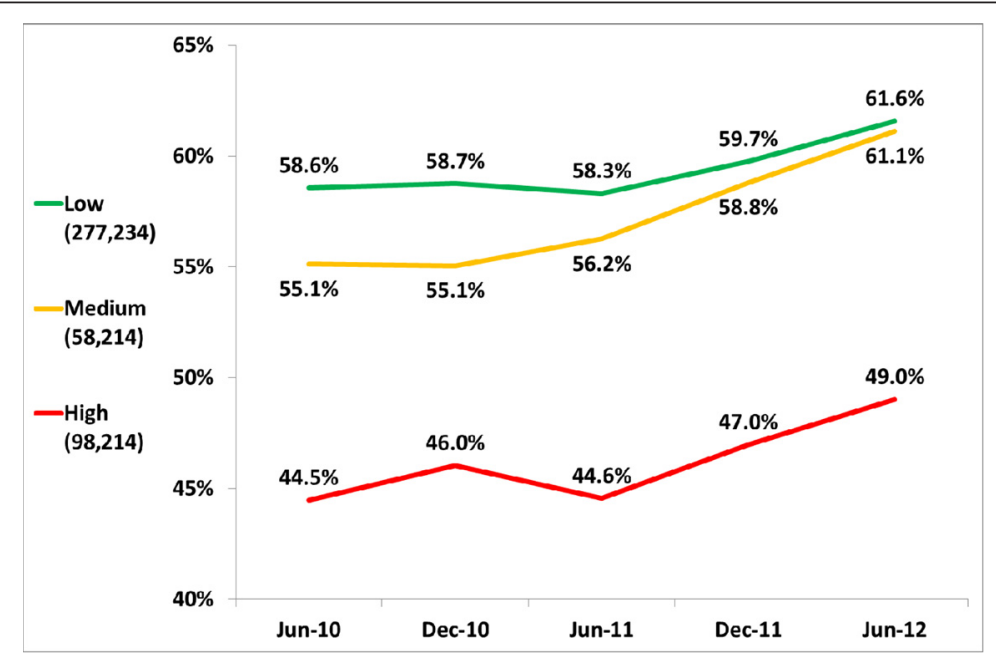

\begin{tabular}{|l|c|c|c|c|c|}
\hline \multicolumn{1}{|c|}{ P-values } & Overall & $\begin{array}{c}\mathbf{0 6 / 2 0 1 0} \\
\mathbf{1 2 / 2 0 1 0}\end{array}$ & $\begin{array}{c}\mathbf{0 6 / 2 0 1 0} \\
\mathbf{0 6 / 2 0 1 1}\end{array}$ & $\begin{array}{c}\mathbf{0 6 / 2 0 1 0} \\
\mathbf{1 2 / 2 0 1 1}\end{array}$ & $\begin{array}{c}\mathbf{0 6} / 2010- \\
\mathbf{0 6} / 2012\end{array}$ \\
\hline Low & $<0.001$ & 0.039 & 0.056 & $<0.001$ & $<0.001$ \\
\hline Medium & $<0.001$ & 0.762 & $<0.001$ & $<0.001$ & $<0.001$ \\
\hline High & $<0.001$ & $<0.001$ & 0.547 & $<0.001$ & $<0.001$ \\
\hline
\end{tabular}

Figure 1 The proportion of patients, stratified by cardiovascular risk who achieved ATP III LDL cholesterol targets during a two-year period (program launched on January 1, 2011). 


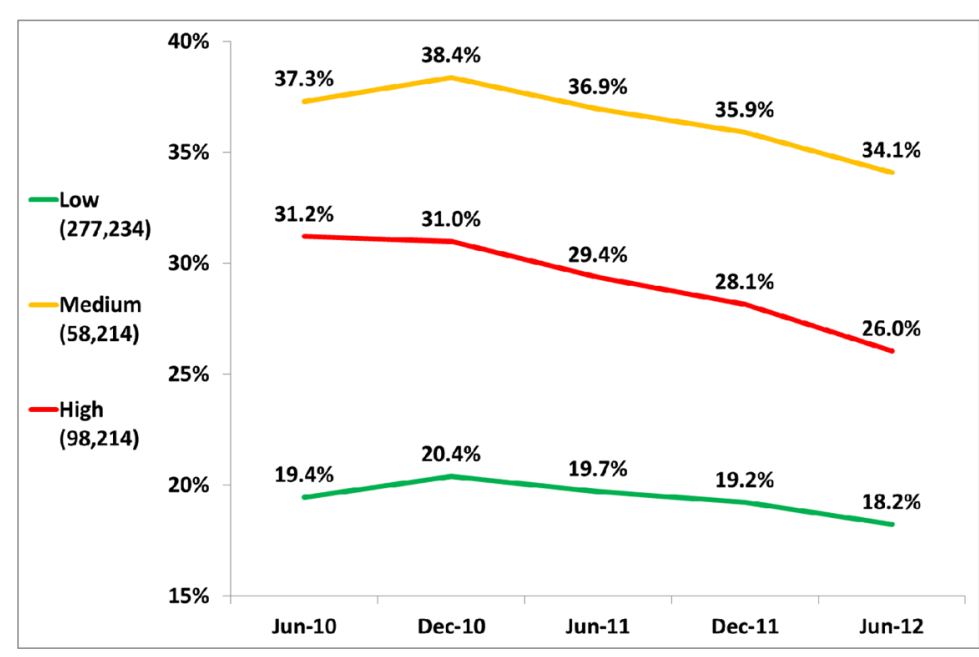

\begin{tabular}{|l|c|c|c|c|c|}
\hline \multicolumn{1}{|c|}{ P-values } & Overall & $\begin{array}{c}\mathbf{0 6 / 2 0 1 0} \\
\mathbf{1 2 / 2 0 1 0}\end{array}$ & $\begin{array}{c}\mathbf{0 6 / 2 0 1 0} \\
\mathbf{0 6 / 2 0 1 1}\end{array}$ & $\begin{array}{c}\mathbf{0 6} / \mathbf{2 0 1 0} \\
\mathbf{1 2 / 2 0 1 1}\end{array}$ & $\begin{array}{c}\mathbf{0 6 / 2 0 1 0} \\
\mathbf{0 6} / \mathbf{2 0 1 2}\end{array}$ \\
\hline Low & $<0.001$ & 0.001 & 0.004 & $<0.001$ & $<0.001$ \\
\hline Medium & $<0.001$ & 0.079 & $<0.001$ & $<0.001$ & $<0.001$ \\
\hline High & $<0.001$ & 0.085 & $<0.001$ & $<0.001$ & $<0.001$ \\
\hline
\end{tabular}

Figure 2 Monthly first line statin prescriptions (program launched on January 1, 2011).

requires committed and persistent long term care of large numbers of patients over the course of years, with relatively high estimations of numbers needed to treat (NNT) to prevent a medical event $[16,17]$. Since the patients evaluated in the current study had already been diagnosed with hyperlipidemia based on earlier recommendations, CHS guidelines recommended measuring their lipid levels annually. Moreover, physicians received reminders when opening a patient's computerized file to repeat lipid profile testing if more than a year had passed since previous testing.

The earlier CHS policy of a uniform LDL-C target did not result in improved control of hyperlipidemia, even though identical effective, convenient, medical treatments with minimal side effects were available. Moreover, the goal of reaching this target in $20 \%$ of the hyperlipidemic population could even have an opposite effect, with physicians preferring to focus on patients who seemed easier to treat rather than those in most need of treatment. We found differences in the use of first-line statins, and particularly in the use of potent statins, among patients with different risk levels, even before implementation of the ATP III guideline- based quality indicators. Such deviation from the uniform target established in CHS during that period attests to differences in clinical decision making when targets were not based on established clinical guidelines published in the medical literature. Ongoing updating of quality indicators and clinical targets according to current scientific knowledge is thus especially important, as is assessment of the benefits expected in physician behavior [18].

We observed a lag of six months in the improvement in patients' lipid profiles after the implementation of the new quality indicators. Possible reasons for the time lapse in perceived improvement were: the large population involved, which in some cases comprised up to $15 \%$ of a physician's patients list, and the time required for a physician to identify relevant patients, to evaluate their willingness to a change in treatment, to enact the change, and to conduct a follow-up blood test to verify its effect.

Even at the end of the study period, fewer than half (46.3\%) of the individuals classified with high cardiovascular risk purchased statins. Similarly, less than half (49\%) reached the LDL-C target. Non-interventional studies have shown comparable rates for both statin use and LDL-C target achievement among high risk patients. Data from the United States National Health and Nutritional Examination Survey (NHANES) showed $54.4 \%$ of individuals with diabetes to achieve LDL-C $<100 \mathrm{mg} / \mathrm{dL}$ in 2007-2008 [19]. In a multi-center study of diabetic 


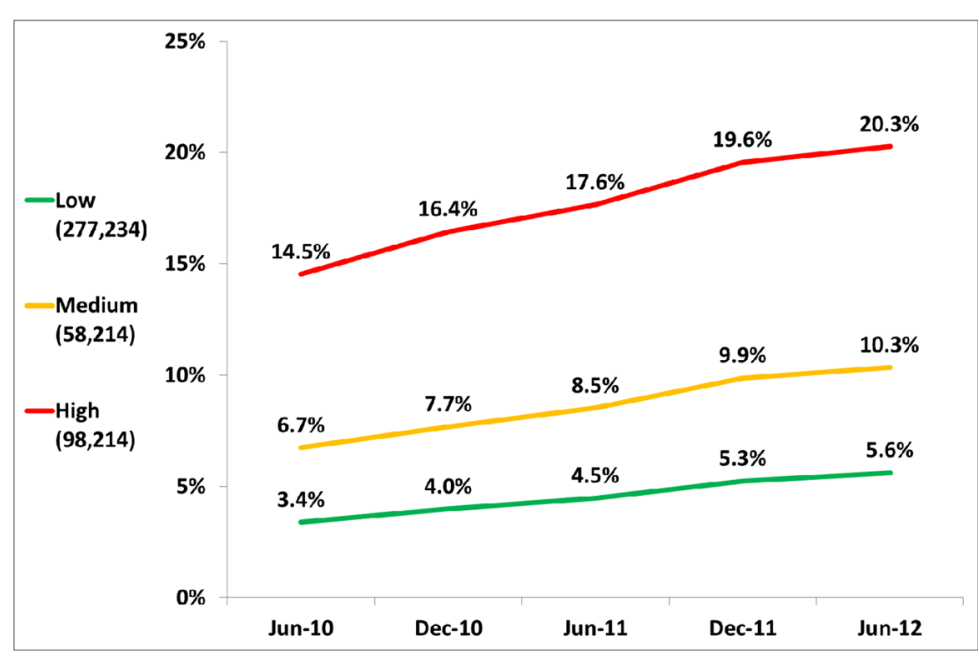

\begin{tabular}{|l|c|c|c|c|c|}
\hline \multicolumn{1}{|c|}{ P-values } & Overall & $\begin{array}{c}\mathbf{0 6 / 2 0 1 0} \\
12 / 2010\end{array}$ & $\begin{array}{c}\mathbf{0 6} / 2010- \\
\mathbf{0 6 / 2 0 1 1}\end{array}$ & $\begin{array}{c}\mathbf{0 6} / \mathbf{2 0 1 0}- \\
\mathbf{1 2 / 2 0 1 1}\end{array}$ & $\begin{array}{c}\mathbf{0 6} / 2010- \\
\mathbf{0 6} / 2012\end{array}$ \\
\hline Low & $<0.001$ & $<0.001$ & $<0.001$ & $<0.001$ & $<0.001$ \\
\hline Medium & $<0.001$ & $<0.001$ & $<0.001$ & $<0.001$ & $<0.001$ \\
\hline High & $<0.001$ & $<0.001$ & $<0.001$ & $<0.001$ & $<0.001$ \\
\hline
\end{tabular}

Figure 3 Monthly potent statin prescriptions (program launched on January 1, 2011).

patients in Korea, only $47 \%$ reached ATP III targets for LDL-C, even though $96 \%$ were taking statins, yet physicians perceived that $71 \%$ achieved the targets [20]. Other Israeli studies found that achievement of LDL-C targets among patients with hyperlipidemia with diabetes or established cardio-vascular diseases is also sub-optimal [21-24]. Achieving LDL-C levels of less than $100 \mathrm{mg} / \mathrm{dl}$ were $65-67 \%$ among patients with coronary disease $[21,23,24], 57 \%$ in diabetic patients [23] and $46.7 \%$ among patients with peripheral vascular disease [24].

Underutilization of lipid-lowering drugs among individuals with diabetes has also been reported in studies conducted in Germany [25] and in China [26]. In a study of individuals who were referred to a lipid clinic, LDL-C targets were reached by only $20 \%$ and $45 \%$ of those not treated and treated with statins, respectively [27].

18 months after change in quality indicators definitions $3 \%, 6 \%$ and $2.9 \%$ more of the hyperlipidemia patients with high, medium and low risk respectively achieved target LDL-C levels. It means additional 1,500, 1,800 and 2,900 patients at the high, medium and low risk groups respectively. Published literature suggests that the effect observed is clinically significant and has the potential to result in a significant reduction in major coronary events [28,29].
A main strength of the current study is the two year follow-up of all patients classified in the same risk categories in a large health maintenance organization. This precludes the selection bias confronting prospective studies that include only physicians and clinics who express willingness to participate $[10,11]$. The current study also contrasts with investigations that assessed target achievement as reduction in cholesterol, without stratification by risk groups [30]. The comprehensive and valid data warehouse of CHS, including drug purchases and laboratory tests is another strength of the study. Perhaps most important, we demonstrated a static situation before the declared implementation of ATP III guideline quality indicators, changes in statin use following the policy change, and increased achievement of LDL-C targets subsequent to the changes in statin use. Still, the study design does not afford conclusions regarding causality; and neither individual patient changes nor adherence of physicians to ATP III guidelines were assessed. Nevertheless, the temporal change observed, first in increased purchases of potent statins, and then in increased rate of LDL-C target achievement, suggests a relationship between these outcomes.

This study has a number of additional limitations. We stratified the patients to three risk groups according to 
the ATP III guidelines. We were unable to retrieve family history of ischemic heart disease or to follow the risk classification calculation on the entire hyperlipidemic population of the CHS so there may be some misclassification in our cohort. But misclassification would be expected to be mainly in the direction of putting patients with high risk into a lower risk group, so the thrust of the PCPs in the program would not be affected. We cannot refute the possibility that factors other than those investigated may have affected clinical decision making and outcomes. No changes in approvals or copayments of relevant drugs occurred during the study period in the face of the expiration of patents on Atorvastatin and Rosuvastatin in Israel at the beginning of the study period. However, it may be that approval policy of potent statins had become more liberal even before the study period, pending the introduction of generics.

We presume that changes in the National Quality Indicators initiative at that period did not have any effect on PCPs behavior in CHS, as treatment goals in the CHS were more ambitious throughout the study period. There were no results of large relevant studies or essentially different clinical guidelines published that could be expected to affect the behavior of the physicians or the general population. The only developments were the notification of physicians of the change in CHS quality indicators regarding treatment of hyperlipidemia, and updates in the reminders in the personal computerized file of patients consistent with the policy change.

\section{Conclusions}

In conclusion, we found that a change in the definition of the quality indicators for the treatment of hyperlipidemia was associated with a change in physician behavior and with improvement in the rate of achievement of target values within 18 months of implementation. The findings suggest that implementation of a quality measure that is tied to established and accepted clinical guidelines, in a setting where physician results are tracked, has the potential to motivate physician behavior and achieve improved clinical results (beyond temporal trends), even in the absence of direct "pay for performance" incentives.

\footnotetext{
Abbreviations

ATP III: Adult Treatment Panel III; CHS: Clalit Health Services; HDL: High density lipoproteins; LDL-C: Low density lipoprotein cholesterol; PCP: Primary care physician; NNT: Numbers needed to treat; NHANES: National Health and Nutritional Examination Survey.
}

\section{Competing interests}

The authors declare that they have no competing interests.

\section{Authors' contributions}

All authors have made substantial contributions to conception and design, involved in drafting the manuscript, read and approved the final manuscript. DC has substantial contribution to data analysis.

\section{Authors' information}

Prof. Shlomo Vinker MD, MHA is a Professor in Family Medicine, and Former Chair of the Department of Family Medicine, at the Sackler School of Medicine, Tel Aviv University, Tel Aviv. He is also Head of the Clinical Quality Indicators program for Clalit Health Services Hospitals and Chairman of the Israeli Association of Family Physicians since 2009.

Prof. Haim Bitterman MD is a Professor of Medicine at the Technion - Israel Institute of Technology. He is the Former Chairman of Medicine, Carmel Medical Center, Haifa and the Former Chairman of the Israel Association of Internal Medicine. He is currently - Chief Physician, Clalit Health Services. Doron Comaneshter MHA, PhD. He is currently - Epidemiologist and Statistician at the Chief Physician Office, Clalit Health Services. Arnon D. Cohen MD,MPH, PhD is the head of the Department of Quality Measurement and Research in the Chief Physician's Office at Clalit Health Services headquarters. Prof. Cohen is an associate professor at Ben-Gurion University. Prof. Cohen formerly served as the medical director of the southern Negev administration of Clalit Health Services.

\section{Author details}

'Chief Physician Office, Central Headquarter, Clalit Health Services, Tel Aviv, Israel. ${ }^{2}$ Department of Family Medicine, Sackler School of Medicine, Tel Aviv University, Tel Aviv, Israel. ${ }^{3}$ Siaal Family Medicine and Primary Care Research Center, Faculty of Health Sciences, Ben-Gurion University of the Negev, Beer-Sheva, Israel.

Received: 15 September 2014 Accepted: 26 March 2015

Published online: 01 June 2015

\section{References}

1. Rosenthal MB. Beyond pay for performance-emerging models of provider-payment reform. N Engl J Med. 2008;359(12):1197-200.

2. Sutton M, Nikolova S, Boaden R, Lester H, McDonald R, Roland M. Reduced mortality with hospital pay for performance in England. N Engl J Med. 2012;367:1821-8.

3. Werner RM, Kolstad JT, Stuart EA, Polsky D. The effect of pay-for-performance in hospitals: lessons for quality improvement. Health Aff (Millwood). 2011;30:690-8.

4. Ryan AM, Blustein J, Casalino LP. Medicare's flagship test of pay-for-performance did not spur more rapid quality improvement among low-performing hospitals. Health Aff (Millwood). 2012;31:797-805.

5. de Bruin SR, Baan CA, Struijs JN. Pay-for-performance in disease management: a systematic review of the literature. BMC Health Serv Res. 2011;11:272.

6. National Cholesterol Education Program (NCEP) Expert Panel on Detection, Evaluation, and Treatment of High Blood Cholesterol in Adults (Adult Treatment Panel III). Third Report of the National Cholesterol Education Program (NCEP) Expert Panel on Detection, Evaluation, and Treatment of High Blood Cholesterol in Adults (Adult Treatment Panel III) final report. Circulation. 2002;106(25):3143-1421.

7. Pletcher MJ, Lazar L, Bibbins-Domingo K, Moran A, Rodondi N, Coxson P, et al. Comparing impact and cost-effectiveness of primary prevention strategies for lipid-lowering. Ann Intern Med. 2009;150(4):243-54.

8. Barham AH, Goff Jr DC, Chen H, Balasubramanyam A, Rosenberger E, Bonds DE, et al. Appropriateness of cholesterol management in primary care by sex and level of cardiovascular risk. Prev Cardiol. 2009;12(2):95-101.

9. Lee JA, Sunwoo S, Kim YS, Oh HJ, Kang HC, Park KC, et al. Achieving recommended low density lipoprotein cholesterol goals and the factors associated with target achievement of hypercholesterolemia patients with rosuvastatin in primary care. Curr Med Res Opin. 2013;29(7):751-60.

10. Bertoni AG, Bonds DE, Chen H, Hogan P, Crago L, Rosenberger E, et al. Impact of a multifaceted intervention on cholesterol management in primary care practices: guideline adherence for heart health randomized trial. Arch Intern Med. 2009;169(7):678-86.

11. Eaton CB, Parker DR, Borkan J, McMurray J, Roberts MB, Lu B, et al. Translating cholesterol guidelines into primary care practice: a multimodal cluster randomized trial. Ann Fam Med. 2011;9(6):528-37.

12. Cabana MD, Rand CS, Powe NR, Wu AW, Wilson MH, Abboud PA, et al. Why don't physicians follow clinical practice guidelines? A framework for improvement. JAMA. 1999;282(15):1458-65.

13. Dreiher J, Comaneshter DS, Rosenbluth Y, Battat E, Bitterman H, Cohen AD The association between continuity of care in the community and health outcomes: a population-based study. Isr J Health Policy Res. 2012;1(1):21. 
14. Rennert G, Peterburg Y. Prevalence of selected chronic diseases in Israel. IMAJ. 2001;3:404-8.

15. Cohen AD, Dreiher J, Regev-Rosenberg S, Yakovson O, Lieberman N, Goldfracht $\mathrm{M}$, et al. The quality indicators program in Clalit Health Services: the first decade. Harefuah. 2010;149(4):204-9. 265. (Hebrew).

16. Richardson G, van Woerden HC, Morgan L, Edwards R, Harries M, Hancock E, et al. Healthy hearts-a community-based primary prevention programme to reduce coronary heart disease. BMC Cardiovasc Disord. 2008;8:18.

17. Superko HR, King 3rd S. Lipid management to reduce cardiovascular risk: a new strategy is required. Circulation. 2008;117(4):560-8.

18. Grimshaw JM, Thomas RE, MacLennan G, Fraser C, Ramsay CR, Vale L, et al. Effectiveness and efficiency of guideline dissemination and implementation strategies. Health Technol Assess. 2004;8(6):iii--iv. 1-72.

19. Kuznik A, Mardekian J. Trends in utilization of lipid- and blood pressure-lowering agents and goal attainment among the U.S. diabetic population, 1999-2008. Cardiovasc Diabetol. 2011;10:31.

20. Hwang JY, Jung CH, Lee WJ, Park CY, Kim SR, Yoon KH, et al. Low density lipoprotein cholesterol target goal attainment rate and physician perceptions about target goal achievement in Korean patients with diabetes. Diabetes Metab J. 2011;35(6):628-36.

21. Pereg D, Elis A, Neuman Y, Mosseri M, Leader A, Segev D, et al. Lipid control in patients with coronary heart disease treated in primary care or cardiology clinics. J Clin Lipidol. 2013;7(6):637-41.

22. Shalev V, Goldshtein I, Halpern Y, Chodick G. Association between persistence with statin therapy and reduction in low-density lipoprotein cholesterol level: analysis of real-life data from community settings. Pharmacotherapy. 2014;34(1):1-8.

23. Elis A, Chodick G, Heymann AD, Kokia E, Flash S, Lishner M, et al. The achievement of target cholesterol level differs between coronary heart disease and diabetic patients. Eur J Intern Med. 2011;22(3):262-5.

24. Pereg D, Neuman Y, Elis A, Minha S, Mosseri M, Segev D, et al. Comparison of lipid control in patients with coronary versus peripheral artery disease following the first vascular intervention. Am J Cardiol. 2012;110(9):1266-9.

25. Berthold HK, Gouni-Berthold I, Bohm M, Krone W, Bestehorn KP. Patterns and predictors of statin prescription in patients with type 2 diabetes. Cardiovasc Diabetol. 2009;8:25.

26. Ting RZ, Yang $X, Y u L W$, Luk $A O$, Kong AP, Tong PC, et al. Lipid control and use of lipid-regulating drugs for prevention of cardiovascular events in Chinese type 2 diabetic patients: a prospective cohort study. Cardiovasc Diabetol. 2010;9:77.

27. Pirro M, Del Giorno R, Lupattelli G, Mannarino MR, Roscini AR, Covelli D, et al. Cardiovascular risk factors and recommended lipid goals attainment among patients referred in a tertiary care lipid clinic. Eur J Intern Med. 2011:22(4):412-7.

28. Ridker PM, MacFadyen JG, Fonseca FA, Genest J, Gotto AM, Kastelein JJ, et al. Number needed to treat with rosuvastatin to prevent first cardiovascular events and death among men and women with low low-density lipoprotein cholesterol and elevated high-sensitivity C-reactive protein: justification for the use of statins in prevention: an intervention trial evaluating rosuvastatin (JUPITER). Circ Cardiovasc Qual Outcomes. 2009;2(6):616-23.

29. Henley E, Chang L, Hollander S. Treatment of hyperlipidemia. J Fam Pract. 2002:51(4):370-6.

30. Alshamsan R, Lee JT, Majeed A, Netuveli G, Millett C. Effect of a UK pay-for-performance program on ethnic disparities in diabetes outcomes: interrupted time series analysis. Ann Fam Med. 2012;10(3):228-34.

\section{Submit your next manuscript to BioMed Central and take full advantage of:}

- Convenient online submission

- Thorough peer review

- No space constraints or color figure charges

- Immediate publication on acceptance

- Inclusion in PubMed, CAS, Scopus and Google Scholar

- Research which is freely available for redistribution

Submit your manuscript at www.biomedcentral.com/submit 